Salud colectiva y salud pública

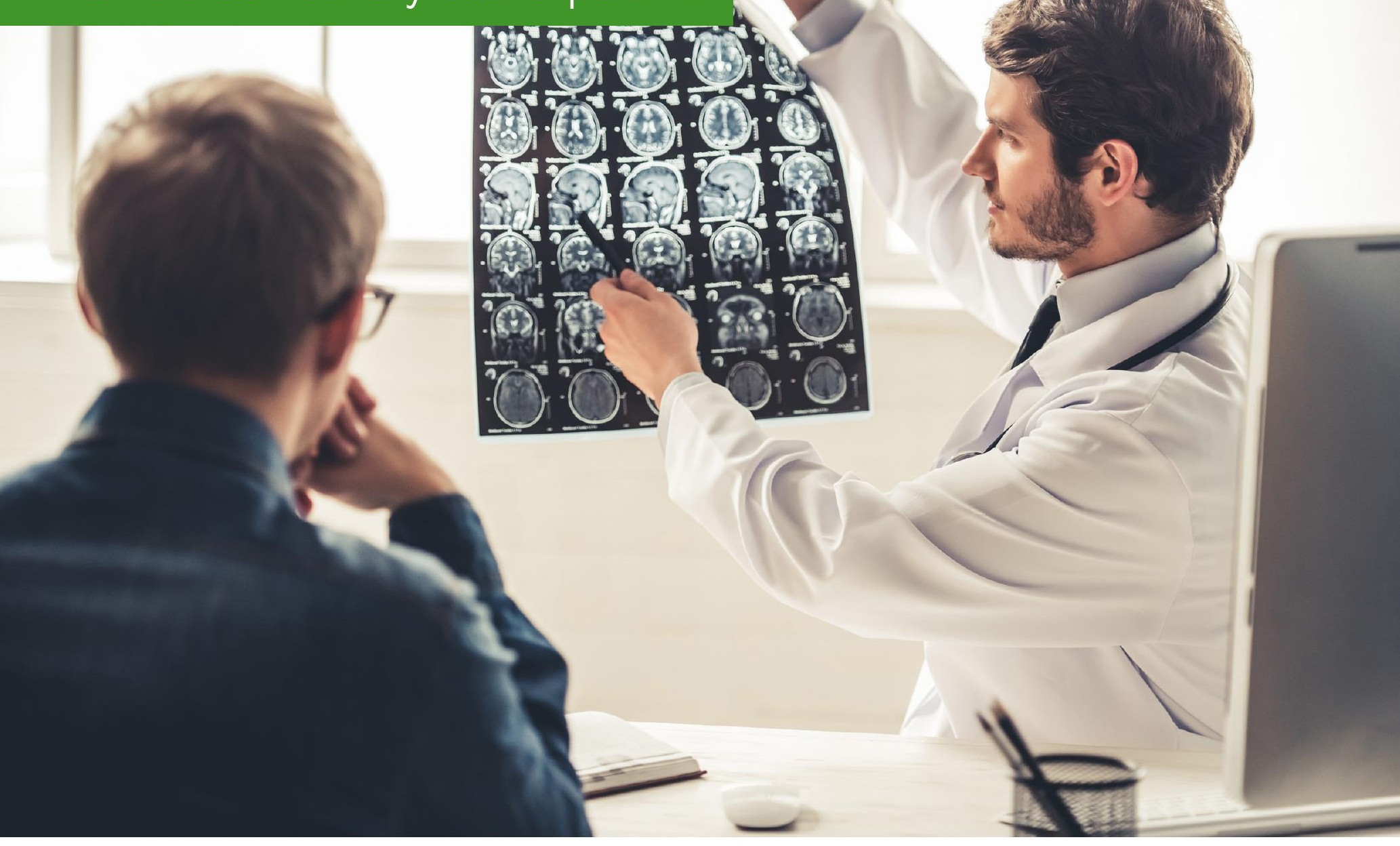

\title{
Salud pública y nuevos derechos individuales a la salud: entre la utopía y la realidad
}

Between Utopia and Reality: Public Health and New Individual Rights to Healthcare

(2) Autor 
Resumen

Abstract

\begin{abstract}
La autonomía es esencial en el ejercicio de la responsabilidad y la capacidad reconocida al usuario en los nuevos derechos de salud. El objetivo es analizar cuál puede ser la influencia del empoderamiento de la autonomía, favorecida por los nuevos derechos sanitarios, en la autorresponsabilidad de profesionales y usuarios ante los problemas de salud pública. Es necesario lograr el autocuidado de los usuarios fomentando sus valores gracias al compromiso de los profesionales como "empoderadores" de los pacientes, además de con mayores cotas de educación en salud y con una ética de la ciudadanía en el uso de los recursos públicos.
\end{abstract}

Autonomy is essential in the exercise of responsibility and the recognized capacity of the user in new health rights. The objective here is to analyze the influence of the empowerment of autonomy arising from new health rights, in the self-responsibility of professionals and users in the face of public health problems. It is necessary to achieve the self-care of users by promoting those values thanks to both the commitment of professionals and by "empowering" patients, with higher levels of health education and an ethic of citizenship in the use of public resources.

Autonomía personal; salud pública; derechos del paciente; autocuidado; responsabilidad social.

Personal autonomy; public health; patient rights; self-care; social responsibility.

Recibido: 16/12/2017. Aceptado: 01/04/2018 
La responsabilidad ante nuestra salud implica escoger o decidir sobre alternativas saludables y correctas en vez de aquellas que envuelven un riesgo para la salud.

La autonomía personal juega un papel esencial en el ejercicio de la responsabilidad y es la autonomía, precisamente, la capacidad esencial que hoy en día se le reconoce al usuario de los servicios sanitarios a través de los nuevos derechos de salud.

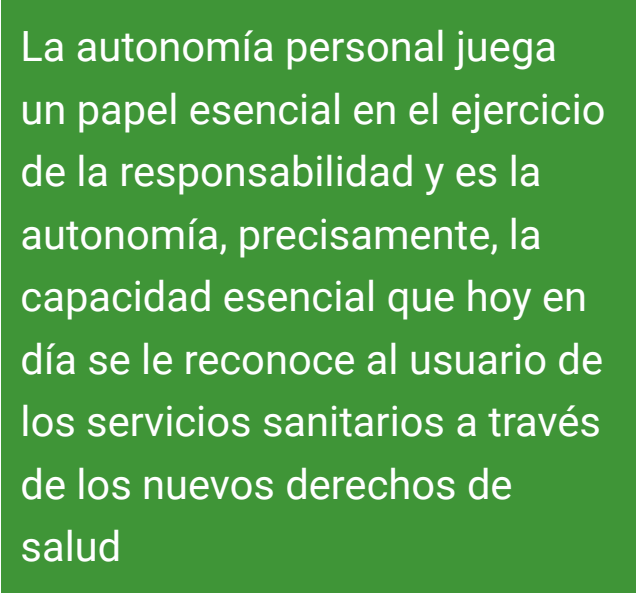

Siguiendo a quienes defienden posiciones moderadas respecto al binomio responsabilidad individual y social en la salud pública (Ferrer, 2003), partimos del presupuesto de que la responsabilidad personal, siendo un valor para promover activamente, no debe utilizarse como criterio de equidad: no debe atenderse equitativamente a los enfermos en función de su responsabilidad, discriminando a los que no la han ejercido correctamente según los parámetros de cada momento. Según M. A. Ortúzar (2016), los estilos de vida son considerados como elecciones voluntarias, que reflejan concepciones de buena vida y sobre las cuales las personas tienen responsabilidades. Pero ¿son los estilos de vida elecciones auténticamente voluntarias? En un esquema ideal de mercado de salud, los igualitaristas rechazarían el sistema de riesgos por considerar que los riesgos de salud no son elegidos, siendo injusto discriminar a las personas por circunstancias relativas a su lotería natural o social. Según A. Puyol (2014), "el fomento de la autorresponsabilidad tiene que ser una de las obligaciones de toda política de salud pública, pero sin absolutizarla, sin abandonar la protección de la salud de los individuos en nombre de su propia responsabilidad".

La salud pública exige además de una responsabilidad personal o individual, una responsabilidad social o colectiva sobre la salud de todos que va más allá de las políticas estrictamente sanitarias y que tiene, entre sus objetivos primordiales, la lucha contra las desigualdades injustas de salud, es decir, aquellas que no tienen una explicación únicamente en la biología y en los estilos de vida voluntarios, y que son evitables (Whitehead, 1992).

Esta responsabilidad colectiva incluye la promulgación de normativa y legislación aplicables en esta materia y en concreto, la que incorpora nuevos derechos sanitarios que empoderan la responsabilidad personal sobre el uso de recursos sanitarios. No obstante, "cualquier sistema de asistencia médica que finalmente se organice no ha de perder de vista que él guarda un carácter subsidiario respecto de esa comunidad primaria que forman el médico, los profesionales sanitarios y el paciente; es esta comunidad, verdadera comunión existencial de personas concretas, la que ha de ser siempre promovida y protegida" (Caponnetto, 2007).

El objetivo de este artículo es analizar desde una perspectiva bioética cuál puede ser la influencia del auge de la autonomía que se ve favorecida en los nuevos derechos, en la autorresponsabilidad de profesionales y usuarios ante los problemas de salud pública. 


\section{La utopía}

Hoy entendemos la salud como "completo bienestar", por lo tanto, definida no solo como integridad o normo-funcionalidad biológica científicamente evaluable sino también, y esta es la mayor novedad, desde la propia subjetividad y desde la propia libertad. Se incorporan al constructo salud unos nuevos elementos definitorios como son los valores, las creencias y las preferencias del individuo.

La utopía sobre la salud como

bienestar se vehicula a través

de nuevos derechos

Esta nueva concepción de la salud exige el reconocimiento y la promoción de unos nuevos derechos que incorporen tanto las nuevas exigencias sociales de autonomía personal como la disponibilidad de unos nuevos medios tecnológicos y de una renovada relación clínica que rompe con el tradicional paternalismo hipocrático. La utopía sobre la salud como bienestar se vehicula a través de nuevos derechos.

Se concibe la salud como una realidad holística que ha tenido un desarrollo histórico que parte de la toma de conciencia de los propios individuos como sujetos de derecho a la libertad personal y a la privacidad, más allá del control de los Estados. Lo que acontece de forma significativa, como es conocido, a partir de la llustración y de las revoluciones americanas y desde ahí, esta toma de conciencia llega al conjunto de nuestra antigua Europa lenta y progresivamente hasta nuestros días.

Una expresión institucional de este proceso es la Declaración Universal de Derechos Humanos desde su primera generación en la que se incluyen la defensa y preservación de la vida, el derecho a la propia integridad y a la salud, el ejercicio de la libertad como base de la propia autonomía: libertad de conciencia y pensamiento, libertad de palabra y reunión, libertad de asociación, libertad religiosa, moral y de conciencia y el derecho a la propiedad.

A partir de aquí se ha continuado y ampliado en las generaciones sucesivas de los Derechos Humanos que incorporan el concepto de la igualdad y el de la solidaridad con una aspiración donde entra en juego una concepción de lo humano más colectiva.

Podemos señalar a los Estados Unidos de Norteamérica como un lugar que ha tenido un papel importante en esta evolución. Allí se han ido dando con claridad y de forma progresiva pasos sucesivos desde la beneficencia médica tradicional, al reconocimiento de los Derechos Humanos individuales. En dichos pasos se significa la reivindicación ante los tribunales de justicia de la autonomía moral del paciente, que posteriormente llega a ser reconocida en las legislaciones positivas proautonomía y es asumida por el colectivo médico en sus declaraciones éticas, hasta llegar a la formulación de la Carta de Derechos del Paciente, ya avanzado el siglo XX.

\section{Crítica de la autonomía como generadora de otros derechos}

Sin embargo, a pesar de la defensa moderna y posmoderna de la autonomía como un valor principal para el ser humano dentro del contexto sanitario, hay que recono- 
cer que el concepto de autonomía moral ha sido comprendido de diferentes formas (Etxeberria, 2002):

- Una de ellas, cuyo autor arquetípico es Mill, es la autonomía como autorrealización. Para esta corriente, la persona autónoma se comprende como la que decide por sí y para sí, desde sus actitudes y sentimientos subjetivos, sin más obligación que no hacer daño directo a la vida o libertad de otros y quedando la solidaridad como opcional. Es un concepto liberal e individualista de la autonomía personal.

- Una segunda, cuyo autor paradigmático es Kant, es la autonomía como autolegislación. La persona autónoma es la que autolegisla para sí misma desde la racionalidad universal de los seres humanos. Presupone la autorrealización de sí mismo que tiene en cuenta lo que es bien para todos.

- Y una tercera, cuyo autor por antonomasia es Taylor, es la autonomía como autenticidad. La persona autónoma es la que es capaz no solo de elegir, sino de elegir lo que construye la propia identidad. Identidad que se construye en diálogo con los valores, los otros, en un marco intersubjetivo y en el contexto social.

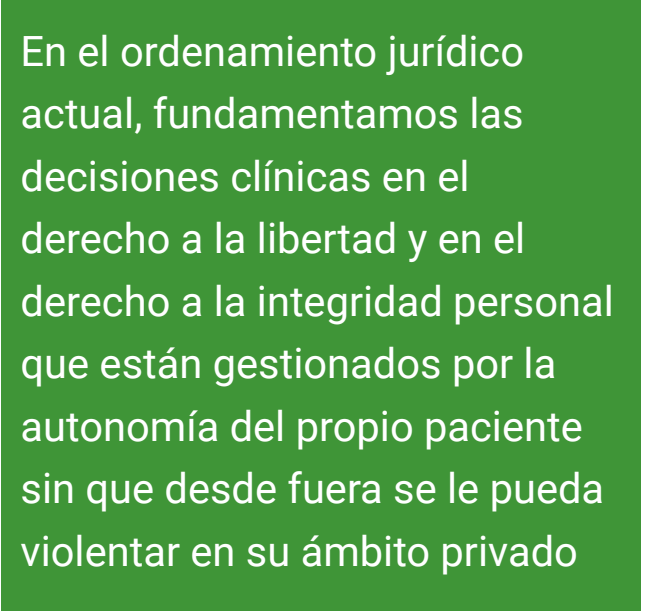

Con este telón de fondo surgen los Principios de la Bioética que han revolucionado la ética médica y para los que un sujeto autónomo es aquel cuya autorregulación está libre tanto de limitaciones externas, como el control y la injerencia de otros, como de limitaciones internas. Una definición que no concreta si la autonomía ha de ejercerse como autorrealización, como autolegislación o como autenticidad. Dejando estas cuestiones morales al ámbito privado del individuo y sin incorporar directamente a la autonomía las necesidades del contexto social donde este se desenvuelve lo que el principialismo americano deja para el principio de justicia.

Por otra parte, en el ordenamiento jurídico actual, fundamentamos las decisiones clínicas en el derecho a la libertad y en el derecho a la integridad personal que están gestionados por la autonomía del propio paciente sin que desde fuera se le pueda violentar en su ámbito privado que incluye su propio organismo, salvo en excepciones muy concretas que se justifican en función del bien colectivo de la sociedad donde se ubica.

Así, a partir del reconocimiento de la autonomía hoy, y para todos y todas, como una cualidad esencial de nuestros tiempos, se reconocen por las legislaciones occidentales los principales derechos de los enfermos, que son:

- Derecho a una asistencia de calidad científica y humana.

- Derecho a recibir una información adecuada, comprensible y veraz.

- Derecho a la autodeterminación y por lo tanto a aceptar o rechazar tratamientos.

- Derecho a la confidencialidad de sus datos y al respeto a su intimidad.

- Derecho a que se les respete su dignidad y se evite el sufrimiento. 
- Derecho a que se le respeten sus convicciones culturales, morales, etc.

Y estos derechos están ampliamente soportados por declaraciones y por normativas jurídicas internacionales y nacionales (en nuestro caso, españolas) como son:

- Declaración Universal de los Derechos Humanos. Asamblea General de las Naciones Unidas, 1948.

- Convenio Europeo sobre Derechos Humanos y Libertades fundamentales, 1950.

- Carta Social Europea, 1961.

- Convenio Internacional sobre los Derechos Civiles y Políticos, 1966.

- Convenio Europeo sobre Derechos Económicos, Sociales y Culturales, 1966.

- Asamblea Parlamentaria del Consejo de Europa. Comisión de Salud y Asuntos Sociales, 1976.

- Plan de Humanización Atención Sanitaria. Carta Derechos y Deberes de Pacientes. Instituto Nacional Salud, 1984.

- Ley General de Sanidad, 1986.

- Derechos de los Pacientes. Asociación Médica Mundial, 1995.

- Convenio relativo a los Derechos Humanos y la biomedicina, 1997.

El profesional sanitario aporta

sus conocimientos técnicos

sobre la salud y el balance

riesgo-beneficio de las

intervenciones diagnósticas y

terapéuticas disponibles. Y el

paciente aporta sus valores,

preferencias y preocupaciones

respecto a su experiencia con la

salud
Como consecuencia de este desarrollo autonomista, la relación clínica entre los profesionales sanitarios y el paciente se ha modificado de forma que cambian los roles, evolucionando de una toma de decisiones unilateral por parte de los profesionales hacia una toma de decisiones compartidas.

En este proceso interactivo:

- El profesional sanitario aporta sus conocimientos técnicos sobre la salud y el balance riesgo-beneficio de las intervenciones diagnósticas y terapéuticas disponibles.

- Y el paciente aporta sus valores, preferencias y preocupaciones respecto a su experiencia con la salud, así como las características que más le importan (beneficios, riesgos y demás implicaciones) de las intervenciones.

$Y$ en este marco entran en juego una serie de nuevos conceptos que reflejan el derecho a la autonomía del paciente como son: equidad; información; consentimiento informado; rechazo de tratamiento; decisiones por sustitución; declaración de voluntad anticipada; intimidad - confidencialidad - privacidad; lex artis - seguridad clínica; limitación de esfuerzo terapéutico. 


\section{La realidad}

Estas posibilidades normativas, que realmente suponen un cambio y un avance importante están actualmente en nuestras manos y han sido el resultado de un proceso reclamado por muchas, pero quiero señalar que no por todas las

Da la sensación de que la normativa sobre la autonomía

y los derechos de los pacientes

llega desde arriba de modo

heterónomo y no se sabe bien

cómo aplicarla, aun hoy, tras

haber transcurrido largos años

de su primera aprobación personas. De tal forma que en los países donde el derecho a la autonomía se ha desarrollado con más fuerza, esta nueva panorámica legal se ha implantado más ampliamente, pero en otros lugares, como son varios países de Europa entre los que incluyo a España, dichos derechos no han sido reclamados explícitamente por la población. Da la sensación de que la normativa sobre la autonomía y los derechos de los pacientes llega desde arriba de modo heterónomo y no se sabe bien cómo aplicarla, aun hoy, tras haber transcurrido largos años de su primera aprobación.

Esta circunstancia, donde hay derechos concedidos por el Estado, muchas veces por trasladar leyes internacionales al ordenamiento jurídico propio, pero que no están reclamados por la población, genera dificultades en la adecuación actual de la relación clínica que están originadas, a mi entender, por diversos factores. Me voy a fijar en los siguientes:

- El primero y más importante es que la incorporación de la autonomía de los pacientes a esta relación es aún hoy claramente contracultural.

- Además, el mismo concepto de autonomía es complejo e incomprendido de forma unívoca.

- En consecuencia con lo anterior, encontramos que los profesionales no saben bien cómo situarse ante la autonomía moral de los usuarios y en realidad, lo más preocupante, al paciente le cuesta asumir un rol activo en el proceso de consentimiento informado.

Voy a comentar brevemente cada uno de estos aspectos.

\subsection{La incorporación de la autonomía es contracultural}

Existe una estrategia de miedo y ocultamiento social de la enfermedad y la muerte, de forma que tenemos una cultura que fomenta poco la adquisición de recursos personales adecuados para afrontar la experiencia de enfermedad propia o la de una persona cercana. Esta situación es cada vez más preocupante pues genera una falta de implicación personal en el cuidado del otro enfermo o persona con discapacidad e incluso, distorsiona el correcto afrontamiento y autocuidado de la propia enfermedad, a diferencia de lo que ocurría en generaciones anteriores.

En este contexto, la relación entre el profesional y su paciente tradicionalmente asimétrica por la diferencia en conocimientos científicos y en la capacidad o poder de gestión de los recursos, tiende hoy a invertirse. 
A pesar de que hasta ahora ha existido, y en bastantes casos sigue existiendo, un enfoque basado en el paternalismo de forma que las decisiones se siguen dejando muchas veces en manos del médico, nos encontramos con situaciones nuevas. Estas se dan cuando, dichas decisiones, se quedan exclusivamente en las manos del propio paciente que utiliza a su médico, más que como el responsable de aportar los conocimientos y las alternativas sobre los remedios efectivos en su caso, como un recurso enciclopédico con quien contrastar lo que ya se piensa que se conoce. Un conocimiento del paciente, al menos parcial, de la propia situación de salud que este alcanza al haberla contrastado previamente con otros médicos, con la información biomédica accesible en internet, con otros profesionales sanitarios o con otras personas que se dedican a ofrecer servicios de salud sin ser sanitarios profesionalmente reconocidos por la ley. La relación clínica se contamina a veces de terceras personas o fuentes de información con las que el usuario contrastará lo que su médico trata de aportarle. Esto genera desconfianza en la relación clínica y rompe el valor de la fidelidad entre médico y paciente.

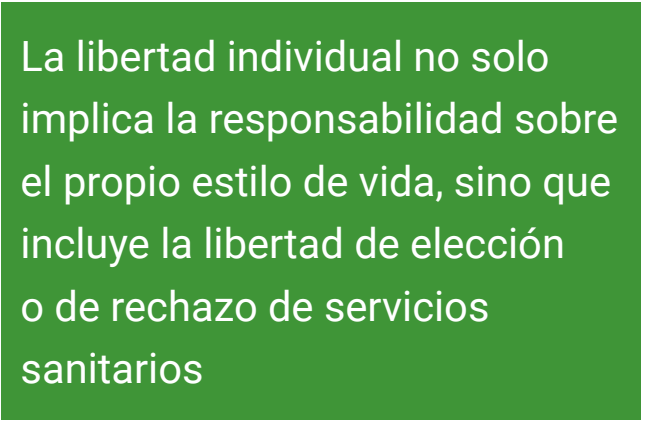

Por otra parte, podemos observar cómo se está dando un progresivo aumento del nivel cultural de la población que contrasta con una escasa educación cívica en relación al uso de los bienes públicos: surge un individualismo exagerado que genera sociedades atomizadas en las que cada uno vela únicamente por su interés particular o corporativo, aunque eso sí, pretendiendo que los recursos que respondan a esta perspectiva particular procedan de los que socialmente se dedican al bien común.

La libertad individual no solo implica la responsabilidad sobre el propio estilo de vida, sino que incluye la libertad de elección o de rechazo de servicios sanitarios.

Tenemos muchos ejemplos de mala utilización de servicios sanitarios. A veces por mala práctica del propio sistema y sus diversos agentes y otras por conductas no responsables de los usuarios.

Brownlee et al. (2017) denuncian que es probable que la sobreutilización perjudique a los pacientes física, psicológica y financieramente, y podría amenazar la viabilidad de los sistemas de atención médica al aumentar los costos y desviar recursos. El uso excesivo, que Chassin y Galvin (1998) definieron como "la provisión de servicios médicos para los cuales el potencial de daño excede el potencial de beneficio", es cada vez más reconocido en todo el mundo. Aunque dicho uso se ha documentado principalmente en países de altos ingresos, los países de ingresos medios y bajos no son inmunes. La evidencia sugiere que el uso excesivo generalizado está ocurriendo en países diversos. La sobreutilización de recursos puede coexistir con necesidades de atención de salud no satisfechas, particularmente en países de bajos ingresos. La simple definición de Chassin y Galvin se complica aún más por la cuestión de qué valores y preferencias deberían determinar el equilibrio entre los beneficios potenciales y los daños aceptables. Ciertamente, diferentes pacientes enfrentados con una opción de tratamientos potencialmente beneficiosos variarán en sus puntos de vista con respecto a las compensaciones de cada uno. Por lo tanto, los valores y las preferencias 
individuales del paciente son fundamentales para definir la atención adecuada para muchas afecciones que se encuentran dentro de la zona gris. Desafortunadamente, los médicos a menudo tienen una comprensión pobre de los valores del paciente, asumiendo incorrectamente en algunos casos que un paciente preferiría evitar la intervención agresiva o invasiva y, en otros casos, que el paciente apreciaría más atención que cuidados. Este mal diagnóstico de preferencia contribuye al uso

Gran parte de la población

que acude a la consulta de

urgencias lo hace para la

atención de problemas no

urgentes y sustituye la atención

del primer nivel con estos

servicios excesivo (y subutilizado) cuando los médicos ofrecen un servicio que es incorrecto para ese paciente individual.

Glasziou et al. (2017) indican cómo la infrautilización (la imposibilidad de prestar un servicio de salud con una alta probabilidad de mejorar la calidad o la cantidad de vida, que sea asequible y que el paciente hubiera deseado) es responsable de una considerable morbilidad y mortalidad evitables. Las causas de la infrautilización son múltiples: acceso inadecuado, fallos en el sistema de salud, que los médicos no sean conscientes o no estén cualificados para proporcionar las intervenciones requeridas y que los pacientes no accedan a ellos o los rechacen. La infrautilización a menudo representa una mala asignación de recursos: las oportunidades para brindar la atención necesaria, efectiva y rentable a menudo compiten con servicios menos efectivos, que pueden ser más comercializados y más costosos. Además, lo que podría representar una subutilización en un país, debe considerarse en el contexto adecuado en otro, de acuerdo con los recursos y las prioridades.

Otro ejemplo es el uso creciente de los servicios de urgencias por problemas no urgentes que supone una preocupación generalizada por dos razones fundamentales: la falta de accesibilidad cultural o administrativa tiende a suplirse con la utilización de los servicios de urgencias y el resultado final supera el gasto que se derivaría de una atención en el primer nivel de atención. Gran parte de la población que acude a la consulta de urgencias lo hace para la atención de problemas no urgentes y sustituye la atención del primer nivel con estos servicios. Esto supone una disminución de la calidad de la asistencia sanitaria en general: pérdida de continuidad asistencial, ausencia de seguimiento de los tratamientos, fallos en la prestación de servicios preventivos y de promoción de la salud. Se acepta que la existencia de una buena interacción médicopaciente disminuye el uso inadecuado del servicio de urgencias y se ha demostrado que la ausencia de provisión de cuidados con el médico regular aumenta el uso del servicio de urgencias por problemas no urgentes, mientras que la continuidad de la asistencia primaria lo reduce (Loría-Castellanos, 2010).

Estas elecciones personales influyen sobre la salud colectiva pues afectan al gasto sanitario. ¿Cómo influyen los nuevos derechos en salud sobre estas elecciones individuales? Están bien basados en el derecho fundamental a la libertad y a la integridad física, pero ¿han de tener límites éticos más allá de los límites legales que ya existen basados en la posibilidad de perjuicio grave y directo a la colectividad por actuaciones individuales irresponsables desde esta perspectiva comunitaria? Esta es una de las cuestiones pendientes y difícilmente regulables, pues queda en manos de una ciudadanía y unas profesiones sanitarias que han de conducirse de modo responsable. 
Posiblemente se sitúa más en el cultivo de una ética cívica basada en la solidaridad y la equidad que en la promulgación de acotamientos legislativos.

\subsection{El mismo concepto de autonomía es complejo e incomprendido de forma unívoca}

Los límites a la autonomía moral de profesionales y ciudadanos no están claros. Observamos diferentes grados de capacidad moral en los ciudadanos, dándose diferentes grados de madurez para el juicio moral, como ya demostró

En realidad, en nuestra

cultura hedonista, cada vez

estamos anímicamente menos

preparados para asumir la

enfermedad
Kohlberg en sus estudios sobre los estadios evolutivos del razonamiento moral y a consecuencia de esto encontramos en las personas, y a veces dependiendo de las circunstancias propias de la diversidad de situaciones, diferentes grados de capacidad de discernimiento ético.

También observamos un desajuste moral manifestado en la negativa a afrontar la enfermedad o al hacerlo mostrando incoherencia con el sistema de valores personales asumido durante el propio recorrido vital. En realidad, en nuestra cultura hedonista, cada vez estamos anímicamente menos preparados para asumirla. Muchas veces se actúa por ansiedad o miedo al sufrimiento, al dolor, la discapacidad o la muerte.

Por estos y otros motivos se dan, a veces, decisiones autónomas que entran en conflicto con otros principios bioéticos como son:

Con el principio de no maleficencia-beneficencia:

- Cuando se piden actuaciones que no corresponden con la indicación médica o lo que entendemos por Lex Artis.

Con el principio de justicia:

- Cuando se pretenden actuaciones que rayan la inequidad como puede ser el trato de favor o la solicitud de privilegios no justificados.

- Cuando se descubren conductas socialmente irresponsables, como los malos hábitos de vida, el descuido de la propia salud, el maltrato al medio ambiente.

- Cuando se dan solicitudes más allá de la cartera de servicios y prestaciones, que no se han considerado dentro de lo financieramente razonable por los servicios públicos.

Por otra parte, nos podemos encontrar que las personas autónomas pueden realizar acciones no autónomas y los considerados a priori como no autónomos pueden realizar en ocasiones acciones autónomas. Ante esto, cabe que nos preguntemos si las decisiones de los ciudadanos deberían ser siempre completamente autónomas o hemos de conformarnos con decisiones suficientemente autónomas.

Para resolver esta cuestión, Faden y Beauchamp (1986) han postulado que la acción autónoma posee tres características básicas con las que nos conformamos, dándolas por suficientes, siguiendo los presupuestos de la bioética norteamericana y que son: la 
correcta intencionalidad; el conocimiento adecuado o suficiente sobre la naturaleza de la acción y sus consecuencias; la ausencia de constricción, sin que exista persuasión inadecuada, ni manipulación y sin coacción.

Y estas premisas son las que estamos aplicando en la toma de decisiones clínicas. Hemos de pensar si son realmente suficientes o cabría incluir alguna más que hiciera referencia a las consecuencias sociales de las decisiones personales.

\subsection{Los profesionales no saben bien cómo situarse ante la autonomía moral de los usuarios y al paciente le cuesta asumir un rol activo en el proceso de consentimiento informado}

\section{Cabe que nos preguntemos \\ si las decisiones de los \\ ciudadanos deberían ser \\ siempre completamente \\ autónomas o hemos de \\ conformarnos con decisiones \\ suficientemente autónomas}

Algunos ejemplos recogidos de la observación directa de la práctica asistencial que ilustran esta afirmación son:

La familia suele ser sistemáticamente y de forma acrítica, la primera destinataria de la información clínica por parte de los profesionales antes que el enfermo. Esta conducta es concordante con la cultura latina, donde la familia juega un papel de agente protector de cada uno de sus miembros y, en principio, puede pensarse que defiende sus intereses. Sin embargo, esta priorización de la familia sin una justificación adecuada contraviene la ley de autonomía del paciente que afirma que el titular de la información es el propio paciente quedando la familia en un segundo plano.

No se suele ofrecer información directa al paciente de un pronóstico de muerte a plazo limitado. Sí se informa del diagnóstico de gravedad, pero no del mal pronóstico. Al menos al propio paciente, aunque sí se haga con la familia.

Existe una mala práctica sobre el consentimiento informado. Se han constatado deficiencias en los procesos de información: tiempo escaso, malas habilidades comunicativas, falta de un lenguaje inteligible, firma de documentos sin lectura previa, firma de documentos por sustitutos o representantes legales sin una justificación, decisiones por sustitución que no aciertan con la voluntad del paciente.

Se reconocen carencias en la Declaración de Voluntades Anticipadas: lo más frecuente es su inexistencia; no se consulta rutinariamente el registro por parte de los profesionales; cuando existen instrucciones previas puede ser que no se verifique una adecuada correspondencia entre lo expresado en la declaración con la situación clínica encontrada.

En el contexto de la relación clínica se da la inequidad: la equidad es un concepto que se relaciona en muchas ocasiones con la mejor o peor distribución que se logre de los recursos sanitarios disponibles, pero también existen inequidades que no tienen su origen en una deficiente distribución económica, sino que se da por una deficiente relación clínica. Por ello es necesario identificar las situaciones y las características personales y sociales de los pacientes que pueden condicionar un mayor riesgo de inequidad 
en la consulta. Por ejemplo, la edad, la pertenencia a un grupo étnico, el género, la orientación sexual, la condición de inmigrante o desplazado, la privación de libertad, la discapacidad o la enfermedad mental, continúan siendo rasgos de exclusión.

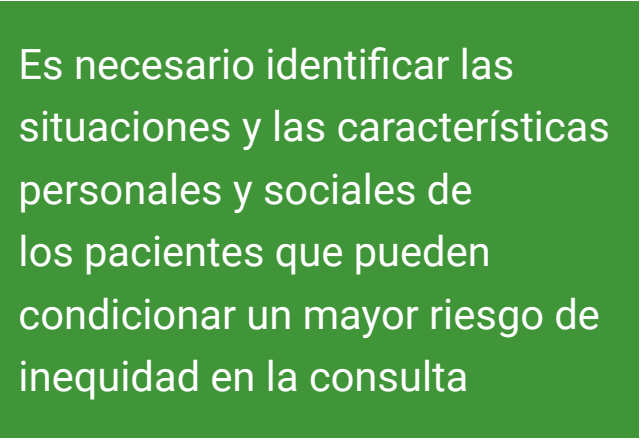

La relación clínica se comprende como una relación de consumo. Así es necesario señalar que a veces se fomenta una tensión entre una organización diseñada para atender enfermos de forma gratuita y universal y una concepción del paciente como cliente o consumidor, lo que estimula al cliente a consumir y a ser el que tome la iniciativa de dicho consumo, sin tener en cuenta otras consideraciones que tienen que ver con el carácter público de los recursos que desea utilizar según su propio criterio.

Estas son razones relevantes por las que la relación clínica se está dificultando. Por ello, debemos preguntarnos cómo debe articularse la nueva relación clínica en este ámbito. Cómo superar los dos extremos que pueden presentarse: el de un médico gestor de las pretensiones de un paciente omnisciente, en un entorno de medios limitados y acuciantes consideraciones de justicia; o por el contrario desde el binomio médico omnisciente y paciente alienado a disposición de las decisiones del profesional.

\section{Estrategias}

Tras realizar un análisis de las dificultades que podemos encontrarnos hoy en la relación clínica, podemos señalar algunas estrategias que pueden ayudar a equilibrar esta relación respetando el derecho de autonomía de los ciudadanos usuarios del sistema y que son:

1. Desarrollar un nuevo profesionalismo y el concepto y praxis de la interprofesionalidad orientada a una atención integral de la salud que tenga en consideración una actualización del concepto de agente social del profesional de la salud, el equilibrio entre la especialización y la coordinación de servicios, la integración holística de la aportación de los diversos profesionales en beneficio armónico del usuario (Rivas y Rivas, 2016).

2. Por parte de los profesionales: asumir en su pensamiento científico una lógica de la probabilidad en lugar de una lógica del determinismo y utilizar una práctica basada en la evidencia científica, el trabajo con carteras de servicios validadas, evitando los conflictos de intereses que desvían el objetivo final de su servicio público hacia otros fines de carácter privado.

3. Desarrollar en los profesionales la capacidad de empoderar al paciente, ofreciendo formación en habilidades de interrelación personal como: la buena comunicación y acogida personal; la igualdad de trato y acceso equitativo; las habilidades como educador; el soporte para la resiliencia. 
4. Por parte del paciente, asumir su responsabilidad personal y social en cuanto al ejercicio de su autonomía: para esto necesita ser capacitado en su toma de decisiones por los propios profesionales.

Un paciente activo, un paciente
empoderado, es un paciente
con capacidad para decidir,
satisfacer necesidades y
resolver problemas, con
pensamiento crítico y control
sobre su vida y su salud

Empoderar significa apoderar, dar poder y está asociado al proceso de fortalecer los derechos y las capacidades de las personas o las comunidades vulnerables con la asunción de un rol activo del ciudadano respecto de la gestión de su propia salud.

Un paciente activo, un paciente empoderado, es un paciente con capacidad para decidir, satisfacer necesidades y resolver problemas, con pensamiento crítico y control sobre su vida y su salud. Que tiene en cuenta no solo sus propias necesidades personales sino que estas se van a abordar en un contexto de sanidad pública que requiere de una correcta aplicación de la equidad y del uso de recursos y reconocer que las conductas personales inciden sobre las posibilidades colectivas.

\begin{abstract}
En la asistencia sanitaria, el autocuidado o cuidado personal es cualquier acción reguladora del funcionamiento del ser humano que se encuentra bajo el control del propio individuo, realizada de forma deliberada y por iniciativa propia. (Segall y Goldstein, 1998)

El autocuidado es la práctica de actividades que los individuos inician y realizan para el mantenimiento de su propia vida, salud y bienestar. (Denyes, Orem, y Bekel, 2001)
\end{abstract}

La ética del autocuidado incluye todas aquellas acciones de atención para la salud que los individuos efectúan para ellos mismos y para sus familias, incluyendo el mantenimiento de la salud, la prevención de las enfermedades, el autodiagnóstico y el autotratamiento. Implica la experiencia de autoestima y la convicción de que cada uno vale mucho y merece lo mejor de sí para sí mismo. Esa mismidad que requiere de autocuidado para mantener la propia identidad y el logro de la propia vida, el propio proyecto vital. Sin embargo, la ética de sí mismo o del autocuidado no está en contraposición a la ética de la alteridad con una vocación más social. Ya que el yo y el nosotros están íntimamente vinculados, de forma que el nosotros no tiene sentido ni tiene posibilidad de crecimiento y progreso sin el yo y viceversa. Se trata de lograr un adecuado equilibrio entre las necesidades propias y las ajenas y por consiguiente entre el consumo de recursos públicos que me corresponden y el que corresponde a los otros.

Así hemos de lograr una nueva concepción y realización de la equidad, lo que supone un equilibrio entre:

- La adecuada distribución de recursos sanitarios.

- La no discriminación de grupos vulnerables.

- La correcta indicación. 
- La participación activa y responsable de la ciudadanía en la toma de decisiones compartidas.

El ciudadano, en definitiva, ha de tomar mayor responsabilidad sobre su salud. Pero ¿qué significa responsabilizarnos de nuestra salud?

La responsabilidad ante nuestra salud implica:

- Modificar los riesgos mediante cambios en nuestro comportamiento.

- Saber y querer escoger o decidir sobre alternativas saludables o correctas.

Requiere un esfuerzo:

- Para ser consciente de los efectos potenciales peligrosos de hábitos de riesgo.

- Para cambiar o modificar nuestros hábitos negativos arraigados.

En este contexto cobra de nuevo gran importancia la educación para la salud, ya que la principal finalidad de la educación para la salud no es la de evitar la enfermedad, sino la de promover estilos de vida saludables y que los individuos tiendan así al autocuidado, que tenga como propósito la consolidación de ciudadanos virtuosos que se conciban a sí mismos como sujetos de derechos y deberes, pero portadores de una ética de la vivencia y de la educación de ciertas virtudes públicas.

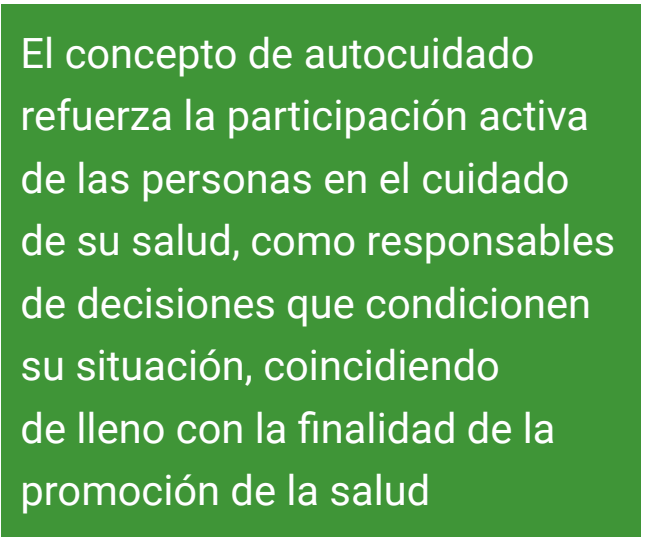

Luis-Gonzalvez (2012) defiende la autorresponsabilidad (en salud) como la acción de

[...] comprender que nuestros actos tienen consecuencias para la salud propia y colectiva. Implica asumir que ética y moralmente tenemos el deber ciudadano de actuar en la vida cotidiana de modo que nuestro comportamiento no resulte nocivo para el mantenimiento de nuestro bienestar ni el de nuestros semejantes. El fundamento ético de este planteamiento es que la reclamación de todo derecho debe acompañarse del insoslayable cumplimiento de un deber.

Una autora desde la Teoría Enfermera que promueve el autocuidado es Dorothea Orem (1993) quien define el objetivo de la enfermería como: "Ayudar al individuo a llevar a cabo y mantener por sí mismo acciones de autocuidado para conservar la salud y la vida, recuperarse de la enfermedad y/o afrontar las consecuencias de dicha enfermedad". Además, afirma que la enfermera puede utilizar cinco métodos de ayuda: actuar compensando déficit, guiar, enseñar, apoyar y proporcionar un entorno para desarroIlo. El concepto de autocuidado refuerza la participación activa de las personas en el cuidado de su salud, como responsables de decisiones que condicionen su situación, coincidiendo de lleno con la finalidad de la promoción de la salud.

Los cuidados enfermeros representan un servicio especializado que se distingue de los otros servicios de salud ofrecidos porque está centrado sobre las personas que no poseen la capacidad para ejercer el autocuidado. Completando así los déficits de autocuidado causados por el desequilibrio entre salud y enfermedad (Orem, 1993). 


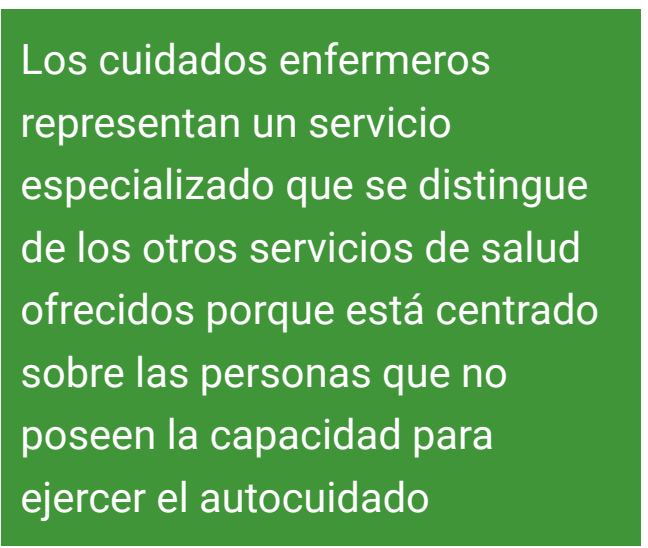

El rol de la profesión enfermera, pues, consiste en persuadir a la persona a avanzar en el camino para conseguir responsabilizarse de sus autocuidados utilizando cinco modos de asistencia: actuar, guiar, apoyar, procurar un entorno que favorezca el desarrollo de la persona y enseñar. Para ello el profesional de enfermería se vale de tres modos de actuación: sistema de intervención totalmente compensatorio, parcialmente compensatorio o de asistencia/enseñanza según la capacidad y la voluntad de las personas.

Por su parte, las actitudes cívicas que tendrían que desarrollar los ciudadanos giran en torno a tres valores básicos:

- Responsabilidad.

- Tolerancia.

- Solidaridad.

Para concluir preguntémonos ¿qué retos son los que presenta la sociedad contemporánea a la sanidad, a la ética y al derecho en cuanto al logro de una correcta relación clínica en función de los objetivos de la salud pública?

A mi juicio pueden ser:

1. Procurar la viabilidad de un sistema sanitario público en un entorno de medios limitados.

2. Ajustar el rol de los profesionales a las nuevas circunstancias y demandas de la sociedad sin perder su papel beneficente desde valores como el altruismo y la defensa de la salud. Para ello deberá tener un papel relevante la bioética como elemento dinamizador del cambio profesional y sensibilizador de los derechos del paciente.

3. Garantizar la autonomía de los usuarios del sistema a través de la promoción de valores como responsabilidad, tolerancia y solidaridad. Lo que hemos de conseguir gracias al compromiso de los profesionales como "empoderadores" de los pacientes, además de con mayores cotas de educación ciudadana en salud y con una ética de la ciudadanía en el uso de los recursos públicos.

\section{Bibliografía}

Brownlee, S., Chalkidou, K., Doust, J., Elshaug, A. G., Glasziou, P. Heath I., .., Korenstein, D. (2017). Evidence for overuse of medical services around the world. The Lancet, 390(10090), 156-168. DOI: https://doi. org/10.1016/S0140-6736(16)32585-5

Caponnetto, M. (2007, Julio 12). La salud como parte viva del bien común y su actual desnaturalización. Salud y Bien Común. V Jornadas Nacionales de la SITA Sección Argentina "Cultura, Ciudad Y Persona”, Mar del Plata, Argentina. Recuperado de http://mariocaponnetto.blogspot.com.es/2007/07/salud-y-bien-comn.html 
Chassin, M. R., \& Galvin R. W. (1998). The urgent need to improve health care quality. Institute of Medicine National Roundtable on Health Care Quality. JAMA, (280), 1000-1005.

Denyes, M., Orem, D., \& Bekel, G. (2001). Self-care: a foundational science. Nursing Science Quarterly, 14(1), 48-54. DOI: https://doi.org/10.1177/089431840101400113

Etxeberria, X. (2002). Temas básicos. Ética de las profesiones. Bilbao, España: Desclée de Brouwer.

Faden, R., \& Beauchamp, T. (1986). A History and Theory of Informed Consent. New York, United States: Oxford University Press.

Ferrer, M. (2003). Equidad y justicia en salud. Implicaciones para la bioética. Acta Bioethica, 9(1), 113-126. DOI: https://doi.org/10.4067/S1726-569X2003000100011

Glasziou, P., Straus, S., Brownlee, S., Trevena, L., Dans, L., Guyatt, G.,..., Saini, V. (2017). Evidence for underuse of effective medical services around the world. The Lancet, 390(10090), 169-177. DOI: https://doi. org/10.1016/S0140-6736(16)30946-1

Loría-Castellanos, J., Flores-Maciel, L., Márquez-Ávila, G., y Valladares-Aranda, M. A. (2010). Frecuencia y factores asociados con el uso inadecuado de la consulta de urgencias de un hospital. Cirugía y Cirujanos, (78), 508-514.

Luis Gonzálvez, I. P., Martínez Calvo, S., \& Álvarez Pérez, A. G. (2012 Oct). Community Engagement, Personal Responsibility and Self Help in Cuba's Health System Reform. MEDICC Review, 14(4), 44-47. Recuperado de: http://www. medicc.org/mediccreview/index. php?issue=22\&id=279\&a=va

Orem, D. E. (1993). Modelo de Orem. Conceptos de enfermería en la práctica. Barcelona, España: MassonSalvat Enfermería.

Ortúzar, M. A. (2016). Responsabilidad social vs. responsabilidad individual en salud. Revista Bioética y Derecho, (36), 23-36. DOI: http://dx.doi.org/10.1344/rbd2016.36.15374

Puyol, A. (2014). ¿Quién es el guardián de nuestra propia salud? Responsabilidad individual y social por la salud. Revista Española Salud Pública, (88), 569-580. DOI: https://doi.org/10.4321/S1135-57272014000500003

Rivas Flores, F. J., y Rivas Gayo, B. (2016). Bioética y asistencia sanitaria. Revista Iberoamericana de Bioética, (02), 1-15. DOI: http://dx.doi.org/10.14422/rib.i02.y2016.008

Segall, A., \& Goldstein, J. (1998). Exploring the Correlates of Self Provided Health Care Behaviour. En D. Coburn, A. D'Arcy, \& G. M. Torrance (Eds.), Health and Canadian Society: Sociological Perspectives (pp. 279280). Toronto, Canada: University of Toronto Press.

Whitehead, M. (1992) The concepts and principles of equity and health. International Journal of Health Services, (22), 429-445. DOI: https://doi.org/10.2190/986L-LHQ6-2VTE-YRRN 